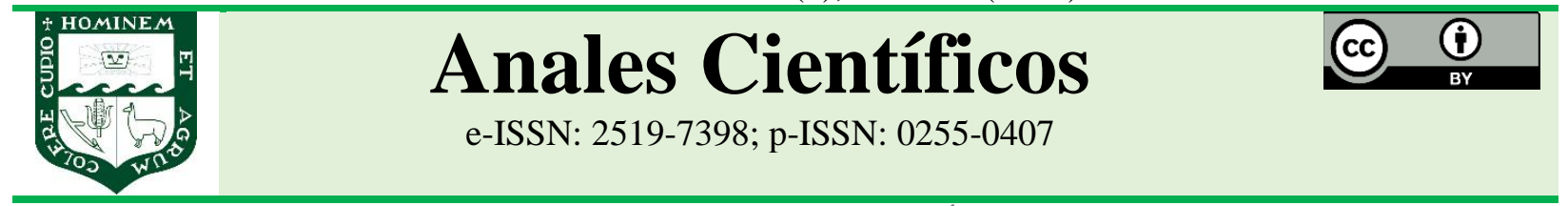

ARTÍCULO ORIGINAL - RESEARCH ARTICLE http://dx.doi.org/10.21704/ac.v82i2.1794

\title{
EFECTO DE LA SUPLEMENTACIÓN ENZIMÁTICA EN PARÁMETROS PRODUCTIVOS DE CUYES (LÍNEA SINTÉTICA P 0.625)
}

\section{Effect of enzymatic supplementation in productive parameters of guinea pigs of (Synthetic Line P 0.625)}

\author{
Ferggie Bernaola Rodriguez ${ }^{1 *(D)}$; Lilia Chauca Francia ${ }^{1}$ (D); Fernando Orrego Vásquez ${ }^{1}$ (D) \\ ${ }^{1}$ Instituto Nacional de Innovación Agraria, La Molina, Lima, Perú. \\ * E-mail: ferggiecbr@hotmail.com
}

Recibido: 02/10/2020; Aceptado: 14/10/2021; Publicado: 31/12/2021

\begin{abstract}
The aim of this study was to evaluate the effect of the supplementation of an enzymatic complex in integral diets for guinea pigs in the growing phase on productive performance. In total, 54 male guinea pigs (Synthetic line $\mathrm{P}$ 0.625 ) were used and submitted to a diet free of the enzyme complex (T1), and two experimental ones, one with $0.1 \%$ (T2) and the other with $0.2 \%$ (T3) of enzyme complex evaluated between two weeks of age (weaning) and after rearing (nine weeks old). It was statically evaluated and there were found no significant difference between treatments in relation to final body weight, weight gain, feed intake and feed conversion; and it was reported the weekly average of percentage of dairy dry matter intake by body weight.
\end{abstract}

Keywords. guinea pigs | diet | enzyme complex | weight

\section{RESUMEN}

El objetivo de la presente investigación fue evaluar el efecto de la suplementación de un complejo enzimático en dietas integrales para cuyes en etapa de recría sobre los parámetros productivos. Se utilizaron 54 cuyes machos de la línea sintética P0.625; sometidos a una dieta libre del complejo enzimático (T1), y dos experimentales una con $0,1 \%$ (T2) y otra con 0,2\% (T3), de complejo enzimático evaluadas entre las dos semanas de edad (destete) y concluida su recría (nueve semanas de edad). Se analizó estadísticamente y no se encontraron diferencias significativas en peso final, ganancia de peso, consumo de alimento y conversión alimenticia; se reportaron los promedios semanales del porcentaje de consumo de materia seca diaria por peso vivo.

Palabras clave: cuyes $\mid$ dieta $\mid$ complejo enzimático | peso

\section{Forma de citar el artículo (Formato APA):}

Bernaola, F., Chauca, L., \& Orrego, F. (2021). Efecto de la suplementación enzimática en parámetros productivos de cuyes (Línea Sintética P 0.625). Anales Científicos. 81(2), 312-317. http://dx.doi.org/10.21704/ac.v82i2.1794

Autor de correspondencia (*): Ferggie Bernaola Rodriguez. Email: ferggiecbr@hotmail.com

(C) Los autores. Publicado por la Universidad Nacional Agraria La Molina.

This is an open access article under the CC BY 


\section{INTRODUCCIÓN}

Existe un creciente interés por alternativas y estrategias alimenticias para la crianza comercial de cuyes (Cavia porcellus) que permitan mejorar los niveles productivos debido a que la alimentación con forraje y residuos de cosecha no llegan a cubrir sus requerimientos nutricionales (Lozada, 2008). Además, los productores de cuyes a nivel comercial están afrontando el problema de falta de forraje por la reducción de áreas de producción forrajera, de modo que vienen adoptando el sistema de alimentación integral (Ccahuana, 2008). El crecimiento de las granjas comerciales se ve limitado por la falta de área para el cultivo de forrajes, por ende, la alternativa del suministro de una ración integral es económicamente viable.

Los aditivos alimenticios cumplen una o varias funciones específicas, habiendo una gran variedad. Entre estos se encuentran los antibióticos, probióticos, enzimas, preservantes, preventivos de daños celular como los antioxidantes, optimizadores de conversión alimenticia como los atractantes, y estimuladores del apetito (Carrillo et al., 2000).

Las enzimas son los catalizadores que permiten la actividad de todas las células del organismo, controlando así todas las funciones de mantenimiento, crecimiento y reproducción de los animales (Carlón, 2007). Las enzimas contribuyen a mejorar la digestibilidad de un nutriente, logrando una digestión eficaz y completa, con lo que mejora sustancialmente la absorción del nutriente (Leiva, 2011). Las enzimas exógenas son utilizadas en la alimentación con dos objetivos bien definidos: complementar las enzimas producidas por el propio animal y proporcionar enzimas que no se consiguen sintetizar (Fischer et al., 2002).

Dentro de los productos ofertados como aditivos en alimentación animal se encuentran los complejos enzimáticos, desarrollados en base a la fermentación con un solo organismo, que genera diversas enzimas totalmente compatibles entre sí. Este tipo de productos se acerca más al requerimiento del nutricionista, ya que suelen incluirse sustratos específicos durante el proceso de fermentación que induce la formación de las enzimas que interesan al productor. Dependiendo de la cepa, la inclusión de almidón induce la formación de amilasas y proteasas (Rodríguez, 2016). El uso de un complejo enzimático en dietas de monogástricos (aves y porcinos) reporta mejoras en la digestibilidad de la proteína vegetal (Alltech, 2010). El presente estudio tuvo como objetivo determinar el efecto de la inclusión de un complejo enzimático en la dieta de cuyes (monogástrico herbívoro) en crecimiento, criados bajo un sistema de alimentación integral, medido a través de la ganancia de peso, consumo de alimento, conversión alimenticia y consumo de materia seca por peso vivo.

\section{MATERIALES Y MÉTODOS}

\section{Localización del experimento.}

La investigación fue conducida en las instalaciones del Programa Nacional de Cuyes del Instituto Nacional de Innovación Agraria (INIA), ubicado en el distrito de La Molina, Lima, Perú, durante la estación de primavera del año 2016. localizada geográficamente en las coordenadas $12^{\circ} 04^{\prime} 25,9^{\prime \prime} \mathrm{S} 76^{\circ} 56^{\prime} 41,9^{\prime \prime} \mathrm{W}$ a una altitud de $241 \mathrm{msnm}$, con temperatura ambiental registrada entre 25 y $28{ }^{\circ} \mathrm{C}$ y humedad relativa del $68 \%$.

\section{Diseño experimental y análisis estadístico.}

Se utilizaron 54 cuyes machos destetados de la Línea Sintética, interracial 5/8 de la raza Perú, de $14 \pm 3$ días de edad, con un peso vivo inicial promedio de $285,9 \pm 10,9$ gramos, que se distribuyen aleatoriamente en unidades experimentales de tres animales/poza, dando un total de 18 unidades experimentales, se utilizó un diseño de bloques completamente aleatorizados (DBCA), con tres tratamientos, tres bloques y dos repeticiones, donde los tratamientos eran el nivel de complejo enzimático en la dieta y los bloques la semana de inicio de evaluación, el diseño de bloques permite anular los efecto de factor externo y que están directamente relacionados a la variable respuesta. Para el análisis de medias se utilizó la prueba de Duncan, la cual no precisa de que el valor $\mathrm{F}$ sea significativo para usarla.

\section{Manejo y alimentación de los animales.}

La infraestructura utilizada fue un galpón tipo costa, los cuyes fueron alojados en pozas, disponiendo de $0,28 \mathrm{~m}^{2} /$ animal, provistas de comedero y bebedero. El 
sistema de alimentación fue integral, donde el suministro de concentrado y agua fue ad libitum. Se utilizó una dieta experimental (Tabla 1) la cual cumple con los estándares nutricionales del NRC (1995). A la dieta experimental se le adicionaron dos niveles del complejo enzimático (CE): 0,1 y 0,2\%, comparadas con una dieta control $(0 \%$ de $\mathrm{CE})$. La ración contenía 2,8 Mcal/kg de energía digestible, $18 \%$ de proteína cruda y $8 \%$ de fibra cruda.

Tabla 1. Composición porcentual y valor nutricional estimado de la dieta experimental.

\begin{tabular}{lc}
\hline Insumos & $\%$ \\
\hline Subproducto de trigo & 49,00 \\
Torta de soya 47 & 28,00 \\
Maíz amarillo & 20,00 \\
Secuestrante & 0,20 \\
Carbonato de calcio & 2,17 \\
Sal & 0,15 \\
Premezcla de vitaminas & 0,12 \\
Cloruro de colina 60\% & 0,12 \\
Inhibidor de hongos & 0,10 \\
DL Metionina & 0,07 \\
Ácido ascórbico & 0,07 \\
Complejo enzimático & 0,00 \\
\hline Total & 100,0 \\
\hline ED (Mcal/kg) & 2,80 \\
Proteína cruda, \% & 18,00 \\
Fibra cruda, \% & 8,00 \\
Lisina, \% & 0,84 \\
Metionina + cisteína, \% & 0,60 \\
Arginina, \% & 1,20 \\
Treonina, \% & 0,60 \\
Triptófano, \% & 0,18 \\
Ácido ascórbico, mg/100 g & 20,00 \\
\hline
\end{tabular}

El complejo enzimático Allzyme Vegpro contiene proteasas en una cantidad mínima de $3750 \mathrm{HUT} / \mathrm{g}$ (cantidad de enzima que produce un hidrolizado con la misma absorbancia a $275 \mathrm{~nm}$ igual a una solución que contenga $1,10 \mu \mathrm{g} / \mathrm{ml}$ de la tirosina en 0,006 NHCL) y celulasa en una cantidad mínima de 22,5 CMCU/g (actividad de carboximetil celulosa que libera $1 \mu \mathrm{mol}$ de azúcar reductor en un minuto bajo las condiciones del ensayo). Este complejo está compuesto por carbonato de calcio, extracto seco de la fermentación de Aspergillus niger, Trichoderma longibrachiatum y Bacillus subtilis.

La ganancia de peso y el consumo de alimento fue evaluado durante siete semanas (2-9 semanas de edad). Para determinar el peso vivo y la ganancia de peso de los cuyes, se pesaron cada siete días, antes de suministrarles alimento. El consumo voluntario del alimento fue estimado semanalmente durante el período experimental por el método convencional, calculando la diferencia entre el alimento ofrecido y el residuo, se utilizó una balanza digital marca TORREY modelo LEQ-10/20, con sensibilidad de un gramo y capacidad máxima de $10 \mathrm{~kg}$.

\section{RESULTADOS Y DISCUSIÓN}

\section{Peso y ganancia de peso}

En la Tabla 2 se muestran los pesos finales, ganancia de peso total y ganancia de peso/día. No hubo diferencias estadísticas significativas entre tratamientos en los parámetros productivos evaluados $(\mathrm{P}<0.05)$. Los pesos y ganancias de pesos obtenidos en este estudio (Tabla 2, Figura 1 y 2) fueron similares a los reportados para esta edad por otros autores utilizando solamente dietas integrales en animales del mismo material genético que la presente investigación (Torres, 2003; Ccahuana, 2008; Inga, 2008; Altamirano, 2012). Asimismo, trabajos similares con usos de enzimas (Guerra, 2015; Cachignia, 2012) no presentaron diferencia estadística para la variable de peso y ganancia de peso, de la misma forma que la presente investigación. Los pesos semanales en la Figura 1 y la ganancia de peso semanal en la Figura 2.

\section{Consumo de alimento y conversión alimenticia}

El consumo de materia seca fue similar entre tratamientos (Tabla 3). Los niveles de consumo fueron menores a lo reportado por Inga (2008), lo que puede atribuirse al tamaño de camada, variable que no fue tomada en cuenta en la presente investigación. La conversión alimenticia en trabajos similares como el de Guerra (2015) y Sopla (2017) no tuvieron una respuesta positiva por el uso de enzimas en dietas para cuyes en etapa de crecimiento de la misma forma que la presente investigación. 
Tabla 2. Efecto de la suplementación de un complejo enzimático sobre el peso y la ganancia de peso.

\begin{tabular}{lcccc}
\hline & & & Niveles del complejo enzimático & T3 0,2\% \\
\cline { 2 - 5 } & & T1 0\% & T2 0,1\% & $1008^{\mathrm{a}}$ \\
\hline Peso final & $\mathrm{g}$ & $1,018^{\mathrm{a}}$ & $994^{\mathrm{a}}$ & $720^{\mathrm{a}}$ \\
Ganancia de peso total & $\mathrm{g}$ & $744^{\mathrm{a}}$ & $698 \mathrm{a}$ & $14,69^{\mathrm{a}}$ \\
Ganancia de peso/día & $\mathrm{g}$ & $15,18^{\mathrm{a}}$ & $14,24^{\mathrm{a}}$ & ${ }^{2}$ \\
\hline
\end{tabular}

${ }^{a}$ Letras diferentes dentro de filas indican diferencia significativa $(\mathrm{p}<0,05)$.

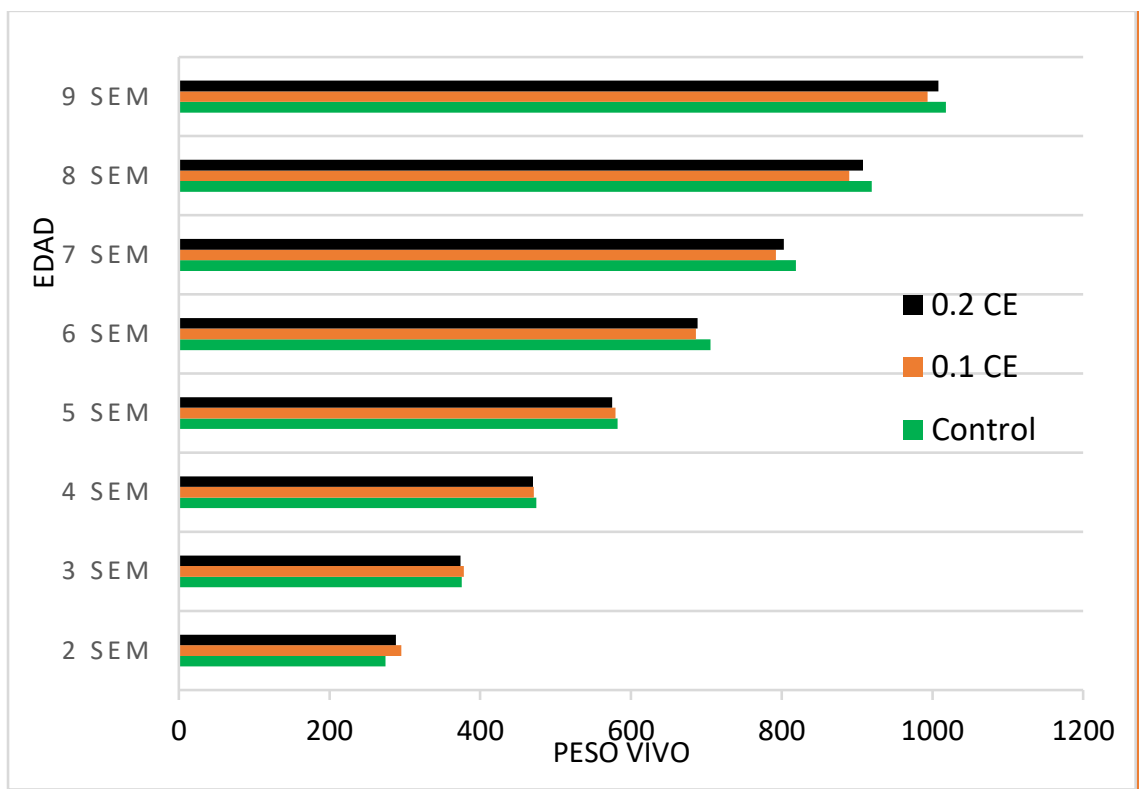

Figura 1. Efecto de la suplementación de un complejo enzimático sobre los pesos semanales.

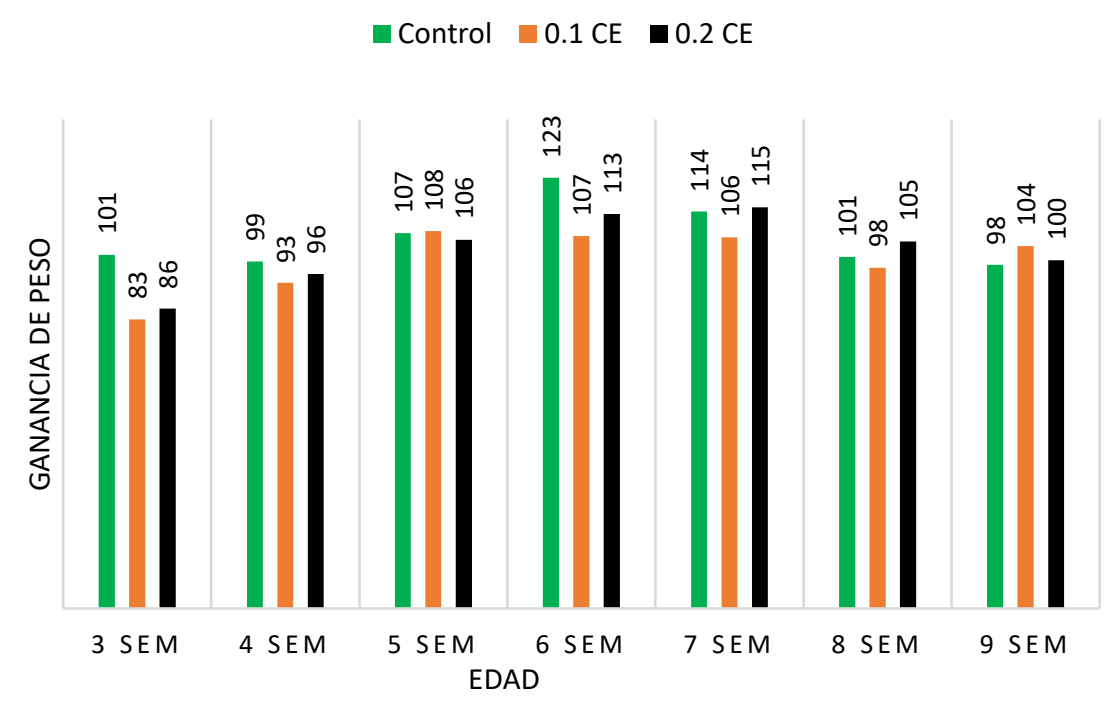

Figura 2. Efecto de la suplementación de un complejo enzimático sobre las ganancias de peso semanales 
A partir de los datos obtenidos de consumo de alimento y peso vivo se determinó tener un indicador porcentual del consumo de materia seca relacionada con el peso vivo (Figura 3). Hoffman y Kester (2013) reconoce la importancia de determinar este parámetro como parte fundamental en los programas de nutrición de ganado. Caballero (1992) observó que hay una relación inversa entre la densidad energética de una ración y el consumo de materia seca por peso vivo del animal, donde ante una dieta de 3,1 y 2,6 Mcal ED/kg el consumo de materia seca era 5,07 y $5,91 \%$ por peso vivo respectivamente. En los resultados de la presente investigación puede apreciarse que, frente a una dieta 2,9 Mcal ED/kg, este parámetro se mantiene en promedio en 6,4; siendo mayor entre la tercera y quinta semana, disminuyendo hasta la novena.

Tabla 3. Efecto de la suplementación de un complejo enzimático sobre el consumo de materia seca y conversión

\begin{tabular}{lccc}
\hline & alimenticia & \\
\cline { 2 - 4 } & & Niveles del complejo enzimático & \\
\cline { 2 - 4 } & T1 0\% & T2 0,1\% & T3 0,2\% \\
\hline Consumo total de MS/cuy (g) & $2146^{\mathrm{a}}$ & $2120^{\mathrm{a}}$ & $2155^{\mathrm{a}}$ \\
Consumo diario de MS/cuy (g) & $43,80^{\mathrm{a}}$ & $43.27^{\mathrm{a}}$ & $43,98^{\mathrm{a}}$ \\
Conversión alimenticia & $2,88^{\mathrm{a}}$ & $3,04^{\mathrm{a}}$ & $2,99^{\mathrm{a}}$ \\
\hline
\end{tabular}

${ }^{\mathrm{a}}$ Letras diferentes dentro de filas indican diferencia significativa $(\mathrm{p}<0,05)$

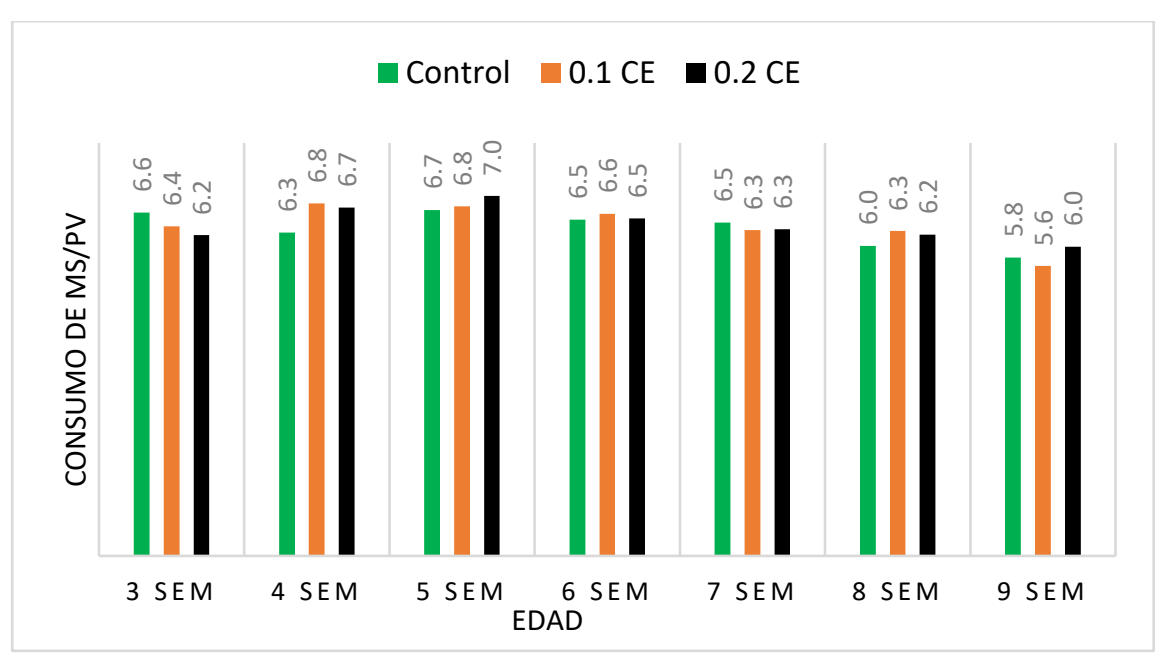

Figura 3. Efecto de la suplementación de un complejo enzimático sobre el promedio semanal del porcentaje de consumo de materia seca diaria por peso vivo.

\section{CONCLUSIONES}

La inclusión del complejo enzimático Allzyme Vegpro en la alimentación integral de cuyes, no contribuye en mejorar los parámetros productivos de cuyes.

\section{Conflictos de intereses}

Los autores firmantes del presente trabajo de investigación declaran no tener ningún potencial conflicto de interés personal o económico con otras personas $\mathrm{u}$ organizaciones que puedan influir indebidamente con el presente manuscrito.

\section{Contribuciones de los autores}

Preparación y ejecución: FB; Desarrollo de la metodología: FB, LC; Concepción y diseño: FB, LC; Edición del artículo: FB, FO; Supervisión del estudio: LC. 


\section{REFERENCIAS}

- Alltech. (2010). Allzyme Vegpro. http://alltech.perulactea.com/2010/05/31/allzymevegpro/

- Altamirano, W. (2012). Efecto de aceite semirefinado de soya en la dieta de cuyes (Cavia porcellus) en crecimiento y engorde. [Tesis de Ingeniero Zootecnista no publicada]. Universidad Nacional Agraria la Molina.

- Caballero, N. (1992). Valor nutricional de panca de maíz: consumo voluntario y digestibilidad en el cuy (Cavia porcellus). [Tesis de Ingeniero Zootecnista no publicada]. Universidad. Nacional Agraria La Molina.

- Cachignia, T. (2012). Probiótico Lactina (Abg2210138) más enzimas (SSF) en dietas Balanceadas a base de palmiste en crecimiento, engorde de cuyes mejorados. [Tesis de Ingeniero Zootecnista, Escuela Superior Politécnica de Chimborazo] Repositorio Institucional - Escuela Superior Politécnica de Chimborazo.

- Carlón, G. (2007). El uso de enzimas en la alimentación de aves. [Tesis de Médico Veterinario y Zootecnista no publicada]. Universidad Michoacana de San Nicolás Hidalgo.

- Carrillo, O., Vega -Villasante, F., Nolasco, H., \& Gallardo, N. (19-22 de noviembre del 2000). Aditivos alimentarios como estimuladores del crecimiento de camarón. V Simposio Internacional de Nutrición Acuícola. Universidad Autónoma de Nuevo León, Yucatán, México.

- Ccahuana, L. (2008). Evaluación del bagazo de marigold en dietas peletizadas con exclusión de forraje verde para cuyes (Cavia porcellus) en crecimiento. [Tesis de Ingeniero Zootecnista no publicada]. Universidad Nacional Agraria la Molina.

- Fischer, G., Maier, J., Rutz, F., \& Bermudez V. (2002). Desempenho de Frangos de Corte Alimentados com Dietas à Base de Milho e Farelo de Soja, com ou sem Adição de Enzimas. Revista Brasileira de Zootecnia, 31(1), 402-410. https://doi.org/10.1590/S151635982002000200015

- Guerra, J. (2015). Evaluación del uso de dietas con tres niveles de enzimas digestivas en la alimentación de cuyes en la fase de crecimiento y finalización. [Tesis de Médico Veterinario Zootecnista, Universidad. Central del Ecuador]. Repositorio Institucional - Universidad Central del Ecuador.

- Hoffman, P., \& Kester, K. (2013). Estimating Dry Matter Intake of Dairy Heifers. http://www.midwestforage.org/pdf/819.pdf.pdf

- Inga, V. (2008). Evaluación de dos niveles de energía digestible y dos niveles de fibra cruda en dietas de crecimiento con exclusión de forraje para cuyes mejorados (Cavia porcellus). [Tesis de Ingeniero Zootecnista no publicada]. Universidad Nacional Agraria la Molina.

- Leiva, G. (2011). Importancia del uso de enzimas en avicultura. Actualidad Porcina. Recuperado el 28 de febrero del 2017de http://www.actualidadporcina.com/battilana/articu los/inportancia-del-uso-enzimas-enavicultura.html

- Lozada, P., Jiménez, R., San Martín, F. y Huamán, A. (2008). Efecto de incluir cebada de grano y/o semilla de girasol en una dieta basada en forraje sobre el momento óptimo económico de beneficio de cobayos en el Valle del Mantaro. Revista de Investigaciones Veterinarias del Perú 24(1):25-31. http://www.scielo.org.pe/scielo.php?script=sci_art text\&pid=S1609-91172013000100003

- Rodríguez-Saldaña, D. (2016). Uso de Enzimas: Consideraciones prácticas y su influencia en los costos de producción del alimento en Ecuador. [Archivo PDF]. https://www.researchgate.net/publication/3266790 17

- Sopla, H. (2017). Utilización de Gallinaza y de un complejo enzimático en la alimentación de cuyes Raza Perú (Cavia porcellus L.) en etapa de recría. [Tesis de Ingeniero Zootecnista, Universidad Nacional Toribio Rodriguez de Mendoza de Amazonas] Repositorio Institucional - Universidad Nacional Toribio Rodríguez de Mendoza de Amazonas.

- Torres, A. (2003). Evaluación de dos niveles de energía y proteína en dietas de crecimiento y engorde para cuyes machos. [Tesis de Ingeniero Zootecnista no publicada]. Universidad Nacional Agraria la Molina. 